\title{
Estimation of the Degree of Anisotropy and Overall Elastic Stiffness of Advanced Anisotropic Materials
}

\author{
Mohamed S. Gaith and Imad Alhayek \\ Department of Mechanical Engineering, Al-Balqa Applied University, Amman, \\ Department of Applied Sciences, Al-Balqa Applied University, Amman
}

Jordan

\section{Introduction}

Most of the elastic materials in engineering are, with acceptable accuracy, considered as anisotropic materials; metal crystals (due to the symmetries of the lattice), fiber-reinforced composites, polycrystalline textured materials, biological tissues, rock structure etc. can be considered as orthotropic materials. In recent years fiber reinforced composite materials have been paid considerable attentions due to the search for materials of light weight, great strength and stiffness. Consequently the determination of their mechanical properties, i.e. stiffness effect, becomes important.

Piezoelectric materials nowadays have been widely used to manufacture various sensors, conductors, actuators, and have been, extensively, applied in electronics, laser, ultrasonics, naval and space navigation as well as biologics, smart structures and many other high-tech areas.

The wide-gap II-VI semiconductors, well known anisotropic materials used in high technology, have received much attention in the past decades since they have important applications in short-wavelength light-emitting diodes (LEDs), laser diodes and optical detectors (Okuyama, 1992). Moreover, semiconductor materials constitute today basic components of emitters and receivers in cellular, satellite, fiberglass communication, solar cells, and photovoltaic systems. Their electronic and structural properties of such systems are subject of considerable interest in nanotechnology as well. For the semiconductor compounds $\mathrm{ZnX}$ and $\mathrm{CdX}(\mathrm{X}=\mathrm{S}$, Se, and Te), the zinc-blend structure (ZB) has the lowest minimum total energy. With respect to classical II- VI semiconductors, these semiconductor compounds have attracted much attention in recent years for their great potential in technological applications (Reich, 2005). They have a high melting point, high thermal conductivity, and large bulk modulus. The hardness and large bulk modulus of these anisotropic materials make them ideal protective coating materials in photovoltaic applications and in machine tools (Reich, 2005). These materials can, therefore, be used for optoelectric devices in which the availability of light sources in the mid-infrared spectral region is crucial for many applications, i. e., molecular spectroscopy and gas-sensor systems for environmental monitoring or medical diagnostics (Leitsmann et al., 2006). Being stable to high temperatures and can be made of sufficiently insulating allows precise measurements 
of piezoelectric, elastic, and dielectric constants. For such data eventually a fully quantummechanical description is essential in order to serve to verify a quantitative theory of piezoelectricity and elasticity in these structures. The window layer compounds CuInSe2, CuInS2, AgGaSe2, and AgGaS2 have a chalcopyrite structure that is closely related those of zinc blend and wurtzite structures. These compounds are found in many applications such as fiberglass communication, thin film solar cells, and photovoltaic systems. Thin film solar cells made from ternary chalcopyrite compounds, such as the aforementioned hetrojunction layers, are characterized by low-cost and clean energy sources. Their high absorption and resistance to deterioration make them good candidate materials for solar cell absorbers. Moreover, due to their flexible optical properties and good stabilities, they are promising compounds for fabricating polycrystalline thin film hetrojunction solar cells (Ramesh et al., 1999; Ramesh et al., 1998; Murthy et al.; 1991). Yet, the significant discrepancy in the efficiency of solar cells between the laboratory scale, over 19\% (Murthy et al.; 1991), and the commercial one, around $13 \%$, is due to the lack of fundamental understanding of interface and junction properties in the film.

Historically, the study of anisotropic elastic materials has been synonymous with study of crystals. For a deep understanding of the physical properties of these anisotropic materials use of tensors is inevitable. Tensors are the most apt mathematical entities to describe direction-dependent-physical properties of solids, and the tensor components characterize physical properties which must be specified without reference to any coordinate system. When all the directions in the material can be considered as equivalent the physical property is isotropic, and consequently, the choice of the reference frame is of no real importance. More often the medium is anisotropic and tensor notation can not be avoided. Specifying the values of the tensor components which represent physical properties of crystals, as Nowack(Nowacki, 1962) points out, do not determine the material constants directly since their values vary with the direction of the coordinate axes. It is, therefore, natural to seek to characterize physical properties of crystals by constants whose values do not depend upon the choice of the coordinate system, i.e. constants which are invariant under all coordinate transformations. Some of such invariants have been obtained using different decomposition methods in the case of photoelastic coefficients ((Srinivasan \& Nigam, 1968)), piezoelectric coefficients (Srinivasan, 1970) and elastic stiffness coefficients (Srinivasan et al. (Srinivasan \& Nigam, 1968; Srinivasan, 1969; Srinivasan, 1985). A physical property is characterized by $\mathrm{n}$ rank tensor that has two kinds of symmetry properties. The first kind is due to an intrinsic symmetry derives from the nature of the physical property itself, and this can be established by the thermodynamical arguments or from the indispensability of some of the quantities involved. The second kind of symmetry is due to the geometric or crystallographic symmetry of the system described.

The purpose of this chapter, hence, is to develop the existing methods of decomposing Cartesian tensors into orthonormal basis using invariant-form to decompose some wellknown tensors into orthonormal tensor basis. Next, as an outcome of these decompositions, to investigate the contributions to the formulation of the physical properties of elastic stress, strain, piezoelectric and elastic stiffness anisotropic materials. Finally, the concept of norm and norm ratios is introduced to measure the overall effect of material properties and to measure the degree of anisotropy. Numerical engineering applications are introduced for several engineering materials like semiconductor compounds, piezoelectric ceramics and fiber reinforced composites. 


\section{Form invariants and orthonormal basis elements}

The decomposition methods of tensors have many applications in different subjects of engineering. In the mechanics of continuous media i.e. in elasticity studies; so far, the stress and strain tensors are decomposed into spherical (hydrostatic) and deviatoric parts, the hydrostatic pressure is connected to the change of volume without change of shape, whereas the change of shape is connected to the deviatoric part of the stress.

The anisotropic elastic properties represented by the fourth rank tensor of elastic coefficients is designated as the elasticity tensor. The constitutive relation for linear anisotropic elasticity is the generalized Hooke's law

$$
\sigma_{\mathrm{ij}}=\mathrm{C}_{\mathrm{ijkm}}{ }^{\varepsilon} \mathrm{km}
$$

which is the most general linear relation between the stress tensor whose components are $\sigma_{\mathrm{ij}}$ and the strain tensor whose components are $\varepsilon_{\mathrm{km}}$. The coefficients of linearity, namely $\mathrm{C}_{\mathrm{ijkm}}$, are the components of the fourth rank elastic stiffness tensor. The elastic properties of crystals appear to be well described in terms of symmetry planes. Symmetry planes (i.e. planes of mirror symmetry) were defined, for example, by Spencer (Spencer, 1983). Cowin et al. (Cowin \& Mehrabadi, 1987) classified the known elastic symmetries of materials and ordered materials on the basis of symmetry planes. Cowin et al. (Cowin \& Mehrabadi, 1987), and Hue and Del Piero(Hue \& Del Piero, 1991) listed ten symmetry classes. There are three important symmetry restrictions on $\mathrm{C}_{\mathrm{ijkm}}$ that are independent of those imposed by material symmetry:

$$
C_{i j k m}=C_{j i k m}, \quad C_{i j k m}=C_{i j m k}, \quad C_{i j k m}=C_{k m i j}
$$

which follow from the symmetry of the stress tensor, the symmetry of the strain tensor, and the thermodynamic requirement that no work be produced by the elastic material in a closed loading cycle, respectively(Srinivasan, 1998; Blinowski, A. \& Rychlewski, 1998). The number of independent components of a fourth rank tensor in three dimensions is 81 , but the restrictions in (2) reduce the number of independents of $C_{i j k m}$ to 21, which corresponds to the most asymmetric elastic solid, namely triclinic media. Since it has 21 independent components, there is considerable information on the material properties apparent a decomposition of $\mathrm{C}_{\mathrm{ij} \mathrm{km}}$ into orthonormal tensor basis would be of interest. The determination of the class system of an elastic medium from its elastic constants in an arbitrary coordinate is not a trivial matter. The problem has been studied thoroughly by several authors (Srinivasan, 1969; Srinivasan, 1985; Spencer, 1983; Cowin \& Mehrabadi, 1987; Hue \& Del Piero, 1991; Srinivasan, 1998; Blinowski \& Rychlewski, 1998; Tu, 1968).

Another interesting material property in anisotropic solids is the direct piezoelectric effect that comprises a group of phenomena in which the mechanical stresses or strains induce in crystals an electric polarization (electric field) proportional to those factors. Besides, the mechanical and electrical quantities are found to be linearly related as following (Srinivasan, 1998). 


$$
P_{i}=d_{i j k} \sigma_{j k}
$$

where $P_{i}$ and $\sigma_{j k}$ denote the components of the electric polarization vector and the components of the mechanical stress tensor, respectively, and $\mathrm{d}_{\mathrm{ijk}}$ are the piezoelectric coefficients forming a third rank tensor. The piezoelectric tensor is a third rank tensor symmetric with respect to the last two indices

$$
\mathrm{d}_{\mathrm{ijk}}=\mathrm{d}_{\mathrm{ikj}}
$$

with 18 coefficients for the noncentrosymmetric triclinic case. Considerable information on the material properties apparent a decomposition of $\mathrm{d}_{\mathrm{ijk}}$ into orthonormal tensor basis would be of interest, as well.

In writing out tensors which represent physical properties of solid materials, it is customary to choose a Cartesian frame reference which has a specific orientation with respect to the material coordinate axes. A physical property is characterized by $\mathrm{n}$ rank tensor that has two kinds of symmetry properties. The first kind is due to an intrinsic symmetry derives from the nature of the physical property itself, and this can be established by the thermodynamical arguments or from the indispensability of some of the quantities involved. The second kind of symmetry is due to the geometric or crystallographic symmetry of the system described.

The symmetry properties of the material may be defined by the group of orthonormal transformations which transform any of these triads into its equivalent positions. For each of the symmetry classes, we will choose as reference system a rectangular Cartesian coordinate system Oxyz, so related to the material directions $v_{1}, v_{2}, v_{3}$ in the material under consideration that the symmetry of the material may be described by one or more of the transformations. Transformations in which the coefficients satisfy the orthogonality relations are called linear orthogonal transformations. In this formulation, the number of elastic constants and their values do not depend on the choice of the coordinate system.

The form-invariant expressions for the electrical susceptibility components, the piezoelectric coefficients and the elastic stiffness coefficients are, respectively

$$
\begin{aligned}
& x_{i j}=v_{a i} v_{b j} A_{a b} \\
& d_{i j k}=v_{a i}{ }^{v_{b j}}{ }^{v_{c k}} A_{a b c} \\
& \mathrm{C}_{\mathrm{ijkm}}=\mathrm{v}_{\mathrm{ai}} \mathrm{v}_{b j} \mathrm{v}^{\mathrm{v}} \mathrm{v}^{\mathrm{v}} \mathrm{dm}{ }^{\mathrm{A}} \mathrm{abcd}
\end{aligned}
$$

Where summation is implied by repeated indices, $v_{a i}$ are the components of the unit vectors $v_{a}(a=1,2,3)$ along the material directions axes. The quantities $A_{a b}, A_{a b c}, A_{a b c d}$ are invariants in the sense that when the Cartesian system is rotated around $O x^{\prime} y^{\prime} z^{\prime}$, where $v_{1}, v_{2}, v_{3}$ form a linearly independent basis in three dimensions but are not necessarily always orthogonal. Their relative orientations in the seven crystal systems are well known (Ikeda, 1990). The corresponding reciprocal triads satisfy the relations 


$$
\mathrm{v}^{\mathrm{ai}} \mathrm{v}_{\mathrm{aj}}=\delta_{\mathrm{ij}}
$$

\subsection{Second rank tensors}

In the theory of linear elasticity, the relation between the stress and strain in a solid body is usually described by Hooke's law which postulates a linear relation between the two. The stress- strain relations for elastic anisotropic material have not been very well established as compared to those of the isotropic material in the classical theory of elasticity. The symmetry properties of the material, due to the geometric or crystallographic symmetry, may be defined by the group of orthonormal transformations which transform any of its triads $v_{\mathrm{a}}$ into its equivalent positions. For the monoclinic symmetric second rank tensor, for instance, the basis elements can be found depending on the form invariant for the monoclinic system. Its form invariant expression, with $v_{2}$ normal to the $v_{1} v_{3}$ plane, can be written as

$$
\sigma_{i j}=A_{11} v_{1 i} v_{1 j}+A_{22} v_{2 i} v_{2 j}+A_{33} v_{3 i} v_{3 j}+A_{31}\left(v_{3 i} v_{1 j}+v_{1 i} v_{3 j}\right)
$$

where $v_{a i}$ are the components of the unit vectors $v_{a}(a=1,2,3)$ along the material directions axes. The corresponding reciprocal triads satisfy the relations (Srinivasan, 1998)

$$
\mathrm{v}^{\mathrm{ai}} \mathrm{v}_{\mathrm{aj}}=\delta_{\mathrm{ij}}
$$

using (8) and orthonormalization by the well known Gram-Schmidt scheme, the four basis elements of the monoclinic system are obtained (Srinivasan, 1998):

$$
\begin{aligned}
& \mathrm{T}_{\mathrm{ij}}^{\mathrm{I}}=\frac{1}{\sqrt{4}} \delta_{\mathrm{ij}} \\
& \mathrm{T}_{\mathrm{ij}}^{\mathrm{II}}=\frac{1}{\sqrt{2}}\left(2 \delta_{i j} \delta_{1 i} \delta_{1 j}+\delta_{3 i} \delta_{3 j}-\delta_{i j}\right) \\
& \mathrm{T}_{\mathrm{ij}}^{\mathrm{III}}=-\frac{1}{\sqrt{6}}\left(3 \delta_{3 i} \delta_{3 j}-\delta_{i j}\right) \\
& \mathrm{T}_{\mathrm{ij}}^{\mathrm{IV}}=\frac{1}{\sqrt{2}}\left(\delta_{3 i} \delta_{1 j}+\delta_{1 i} \delta_{3 j}\right)
\end{aligned}
$$

It is well known that for a symmetric second order tensor is of dimension six; an orthonormal basis set of six elements can be constructed. By taking cyclic permutation of $\{1$, $2,3\}$; the elements V and VI can be generated from IV in (10) as

$$
\begin{aligned}
& \mathrm{T}_{\mathrm{ij}}^{\mathrm{V}}=\frac{1}{\sqrt{2}}\left[\delta_{1 i} \delta_{2 j}+\delta_{2 i} \delta_{1 j}\right] \\
& \mathrm{T}_{\mathrm{ij}}^{\mathrm{VI}}=\frac{1}{\sqrt{2}}\left[\delta_{2 i} \delta_{3 j}+\delta_{3 i} \delta_{2 j}\right]
\end{aligned}
$$

A complete orthonormal basis for the second rank symmetric tensor will be the set $\{\mathrm{I}, \mathrm{II}, \ldots$, $\mathrm{VI}$. The decomposition of $\sigma_{\mathrm{ij}}$ is given in terms of these basis elements as 


$$
\sigma_{i j}=\sum_{k}\left(\sigma, T_{i j}^{k}\right) T_{i j}^{k}, \quad(k=I, I I, \ldots, V I)
$$

where $\left(\sigma, T_{i j}^{k}\right)$ represents the inner product of and the $k^{\text {th }}$ elements, $T_{i j}^{k}$, of the basis. Hence, the second rank symmetric tensor is decomposed into six orthonormal terms expressed in matrix form:

$$
\begin{aligned}
& \sigma_{\mathrm{ij}}=\left[\begin{array}{ccc}
\frac{1}{3} \sigma_{\mathrm{pp}} & 0 & 0 \\
0 & \frac{1}{3} \sigma_{\mathrm{pp}} & 0 \\
0 & 0 & \frac{1}{3} \sigma_{\mathrm{pp}}
\end{array}\right]+\left[\begin{array}{ccc}
\frac{1}{2}\left(2 \sigma_{11}+\sigma_{33}-\sigma_{p p}\right) & 0 & 0 \\
0 & \frac{1}{2}\left(-2 \sigma_{11}-\sigma_{33}+\sigma_{p p}\right) & 0 \\
0 & 0 & 0
\end{array}\right]+ \\
& +\left[\begin{array}{ccc}
\frac{1}{6}\left(-3 \sigma_{33}-\sigma_{p p}\right) & 0 & 0 \\
0 & \frac{1}{6}\left(-3 \sigma_{33}-\sigma_{p p}\right) & 0 \\
0 & 0 & \frac{1}{6}\left(6 \sigma_{33}-2 \sigma_{p p}\right)
\end{array}\right]+\left[\begin{array}{ccc}
0 & 0 & \sigma_{13} \\
0 & 0 & 0 \\
\sigma_{13} & 0 & 0
\end{array}\right]+ \\
& +\left[\begin{array}{ccc}
0 & \sigma_{12} & 0 \\
\sigma_{12} & 0 & 0 \\
0 & 0 & 0
\end{array}\right]+\left[\begin{array}{ccc}
0 & 0 & 0 \\
0 & 0 & \sigma_{23} \\
0 & \sigma_{23} & 0
\end{array}\right]
\end{aligned}
$$

From (13), the second rank symmetric tensor, $\sigma_{i j}$, is decomposed into six terms, each of which has a physical meaning. Also, the second rank symmetric tensor is virtually decomposed into two parts:

$$
\sigma_{i j}=\frac{1}{3} \sigma_{p p} \delta_{i j}+\left(\sigma_{i j}-\frac{1}{3} \sigma_{p p} \delta_{i j}\right)
$$

From (14), it is clear that the symmetric second rank stress tensor is decomposed into spherical (hydrostatic pressure) part, $\frac{1}{3} \sigma_{\mathrm{pp}} \delta_{\mathrm{ij}}$, which is the first term of (13), and the deviatoric part, $\left(\sigma_{i j}-\frac{1}{3} \sigma_{p p} \delta_{i j}\right)$, which is the sum of the other five terms of (13). Hence, it is shown that the method is able to decompose the symmetric second rank stress (and strain, in a similar manner) tensors into the spherical part which is connected to the change of volume without change of shape, and into deviatoric part, which is connected to the change of shape. This result is very well known in the literature. On the other hand, this method is introducing a new form of decomposition, which has a more featured and transparent 
physical information. It is easily verified that the sum of the six decomposed tensors is the symmetric second rank tensor, $\sigma_{\mathrm{ij}}$. Physically, each of the six tensor parts is associated with a distinct type of deformation; the first part of (13) represents the spherical (hydrostatic pressure) effect, the second and third parts represent combined simple extension or contraction along the various symmetry axes. The second term could be, for example, stress of a non-uniform distribution of pure shear stress, which occurs in a long rod subjected to pure torsion, while the last three parts represent simple shearing in the symmetry planes. Besides, the deviatoric part of the stress tensor is decomposed into traceless tensors each of them is related to shearing which represents a general symmetric second rank tensor (stress and strain tensors). The results agree with previous studies considered as special cases of this general case, for instance, Blinowski et al. (Blinowski \& Rychlewski, 1998) have decomposed a tensor of only shear into exactly identical forms to the last three terms of (13) for this specific case.

\subsection{Third rank tensors}

In the continuum approach to the study of anisotropic solids it is well known that certain physical properties can be represented by tensors. The polarization of a crystal produced by an electric field is an example of an anisotropic material property that is represented by tensors. If a stress is applied to certain crystals they develop an electric moment whose magnitude is proportional to the applied stress; known as piezoelectric effect. The piezoelectric effect in materials has not attracted much attention until after the Second World War, since when the applications and the research of piezoelectric materials have advanced greatly. Piezoelectric materials nowadays have been widely used to manufacture various sensors, conductors, actuators, resonators, oscillators and monitors. They also play an important role in the so-called smart structures. In fact, piezoelectric materials have been applied extensively in electronics, laser, ultrasonics, microsonics, naval and space navigation as well as biologics and many other high-tech areas. The piezoelectric coefficients appear in the equation $P_{i}=d_{i j k} \sigma_{j k}$, where $P_{i}$ are the components of the electrical polarization vector and $\sigma_{\mathrm{jk}}$, the components of the stress tensor. The form invariant expressions are derived for many classes of piezomagnatic (Srinivasan, 1970), and piezoelectric coefficients (Tsai, 1992). Although such constitutive equations are form invariant with respect to arbitrary orthogonal coordinate transformations, the coefficients, $\mathrm{d}_{\mathrm{ijk}}$, do not determine directly the material constants since their values vary with the direction of the coordinate axes. The piezoelectric coefficients with the following symmetry $d_{i j k}=d_{i k J}$ the number of nonvanishing independent coefficients is reduced from 27 to 18 independent coefficients for the triclinic system. For the monoclinic system, for example, of class 2, the number of independent coefficients is reduced to 8 , for the orthotropic system of class $\mathrm{mm} 2$ is reduced to five coefficients, and for the hexagonal system of class $6 \mathrm{~mm}$ is reduced to three independent coefficients. The form invariant expression for the hexagonal system class $6 \mathrm{~mm}$ is (Srinivasan, 1970)

$$
d_{i j k}=d_{1} \delta_{3 i} \delta_{3 j} \delta_{3 k}+d_{2}\left(\delta_{3 k} \delta_{i j}+\delta_{3 j} \delta_{i k}\right)+d_{3} \delta_{3 i} \delta_{j k}
$$

Following the same procedure used for second rank tensor, the basis elements are 


$$
\begin{aligned}
& \mathrm{T}_{i j k}^{\mathrm{I}}=\delta_{3 i} \delta_{3 j} \delta_{3 k} \\
& \mathrm{~T}_{i j k}^{\mathrm{II}}=\frac{1}{\sqrt{2}}\left(\delta_{3 i} \delta_{j k}-\delta_{3 i} \delta_{3 j} \delta_{3 k}\right) \\
& \mathrm{T}_{i j k}^{I I I}=\frac{1}{2}\left(\delta_{3 k} \delta_{i j}+\delta_{3 j} \delta_{i k}-2 \delta_{3 i} \delta_{3 j} \delta_{3 k}\right)
\end{aligned}
$$

The decomposition of $d_{i j k}$ is given in terms of this basis elements as

$$
\begin{aligned}
d_{i j k} & =\sum_{k}\left(d, T_{i j k}^{q}\right) T_{i j k}^{q}, \quad(q=I, I I, I I I) \\
& =\left(d, T_{i j k}^{I}\right) T_{i j k}^{I}+\left(d, T_{i j k}^{I I}\right) T_{i j k}^{I I}+\left(d, T_{i j k}^{I I I}\right) T_{i j k}^{I I I}
\end{aligned}
$$

where $\left(d, T_{i j k}^{q}\right)$ represents the inner product of $d_{i j k}$ and $q_{t h}$ elements, $T_{i j k}^{q}$ of the basis .

$$
\begin{aligned}
& \mathrm{d}_{\mathrm{ijk}}=\left[\begin{array}{cccccc}
0 & 0 & 0 & 0 & 0 & 0 \\
0 & 0 & 0 & 0 & 0 & 0 \\
0 & 0 & \mathrm{~d}_{33} & 0 & 0 & 0
\end{array}\right]+\left[\begin{array}{cccccc}
0 & 0 & 0 & 0 & 0 & 0 \\
0 & 0 & 0 & 0 & 0 & 0 \\
\frac{1}{2}\left(\mathrm{~d}_{31}+\mathrm{d}_{32}\right) & \frac{1}{2}\left(\mathrm{~d}_{31}+\mathrm{d}_{32}\right) & 0 & 0 & 0 & 0
\end{array}\right]+ \\
& +\left[\begin{array}{cccccc}
0 & 0 & 0 & \frac{1}{2}\left(\mathrm{~d}_{24}+\mathrm{d}_{15}\right) & 0 & 0 \\
0 & 0 & \frac{1}{2}\left(\mathrm{~d}_{24}+\mathrm{d}_{15}\right) & 0 & 0 & 0 \\
0 & 0 & 0 & 0 & 0 & 0
\end{array}\right]
\end{aligned}
$$

Physically, we have decomposed the tensor $\mathrm{d}_{\mathrm{ijk}}$ into three independent tensors, each has an independent piezoelectric coefficient. If a tensile stress $\sigma_{3}$ is applied parallel to $x_{3}$ which is a diad axis of the crystal, the first matrix in (18) shows that the components of polarization are given by the moduli in the third column of the first matrix. Thus, the decomposition that we present is decomposing the polarization along orthogonal axes into three parts; the first part is the polarization along the diad axes due to normal stress, the second part, the polarization along the nondiad orthogonal axes due to normal stress, and the third part, is the polarization due to the shear stresses.

\subsection{Fourth rank tensors}

Fourth rank tensors were introduced in specification of physical properties for several anisotropic media. A decomposition of these tensors into independent elementary tensors is undertaken, to offer valuable insight into the tensor structure. In an anisotropic material, the 
elasticity symmetric tensor generally contains twenty-one non-zero distinct constants. When the material has some kind of symmetry, the number of these coefficients is reduced if the coordinate axes coincide with symmetry axes for the material.

In analyzing the mechanical properties of anisotropic linear elastic medium, a tensor of fourth rank is required to make up a linear constitutive relation between two symmetric second-rank tensors, each of which represents some directly detectable and measurable effect in the medium. The constitutive relation characterizing linear elastic anisotropic solids is the generalized Hook's law as expressed in (1). Due to the symmetries in (2), the number of elastic coefficients is reduced from 81 to 21 which correspond to the most asymmetric elastic solid, namely, triclinic medium. The presence of symmetry in a medium reduces still further the number of independent elastic coefficients. For example, monoclinic symmetry medium (Tu. 1968) reduces the number of the non-vanishing independent elastic coefficients to 13 , similarly, orthotropic to 9 , hexagonal to 5 , cubic to 3 and isotropic medium (the most symmetry) to 2 elastic coefficients.

The isotropic system has the well defined form invariant as following (Srinivasan, 1968):

$$
C_{i j k m}=A_{1} \delta_{i j} \delta_{k m}+A_{2} \delta_{i k} \delta_{j m}+A_{3} \delta_{i m} \delta_{j k}
$$

where $\mathrm{A}_{1}, \mathrm{~A}_{2}$ and $\mathrm{A}_{3}$ are constants, and later, $\mathrm{Tu}(\mathrm{Tu}$. 1968) has reduced the three tensors into two basis elements. Following the same procedure presented in previous sections, the decomposition of $\mathrm{C}_{\mathrm{ijkm}}$ for the isotropic system is given in terms of the basis elements as:

$$
\begin{aligned}
C_{i j k m} & =\sum_{k}\left(C, T_{i j k m}^{k}\right) T_{i j k m}^{k}, \quad(k=I, I I) \\
& =\left(C, T_{i j k m}^{I}\right) T_{i j k m}^{I}+\left(C, T_{i j k m}^{I I}\right) T_{i j k m}^{I I}
\end{aligned}
$$

where $\left(C, T_{i j k m}^{k}\right)$ represents the inner product of $C_{i j k m}$ and the kth elements, $T_{i j k m}^{k}$, of the basis, and

$$
\begin{aligned}
& \mathrm{T}_{\mathrm{ijkm}}^{\mathrm{I}}=\frac{1}{3} \delta_{i j} \delta_{k m} \\
& \mathrm{~T}_{\mathrm{ijkm}}^{\mathrm{II}}=\frac{1}{6 \sqrt{5}} \delta_{i j} \delta_{\mathrm{km}}\left(3\left(\delta_{i k} \delta_{j m}+\delta_{i m} \delta_{j k}\right)-2 \delta_{i j} \delta_{k m}\right)
\end{aligned}
$$

substituting these elements, then

$$
\begin{aligned}
C_{i j k m}= & \frac{1}{9} C_{p p q q} \delta_{i j} \delta_{k m}+ \\
& \frac{1}{36(5)}\left(6 C_{p q p q}-2 C_{p p q q}\right)\left(3\left(\delta_{i k} \delta_{j m}+\delta_{i m} \delta_{j k}\right)-2 \delta_{i j} \delta_{k m}\right)
\end{aligned}
$$

and in matrix form as: 


$$
C_{p q}=K_{v}\left[\begin{array}{cccccc}
1 & 1 & 1 & 0 & 0 & 0 \\
1 & 1 & 1 & 0 & 0 & 0 \\
1 & 1 & 1 & 0 & 0 & 0 \\
0 & 0 & 0 & 0 & 0 & 0 \\
0 & 0 & 0 & 0 & 0 & 0 \\
0 & 0 & 0 & 0 & 0 & 0
\end{array}\right]+2 G_{v}\left[\begin{array}{cccccc}
\frac{2}{3} & -\frac{1}{3} & -\frac{1}{3} & 0 & 0 & 0 \\
-\frac{1}{3} & \frac{2}{3} & -\frac{1}{3} & 0 & 0 & 0 \\
-\frac{1}{3} & -\frac{1}{3} & \frac{2}{3} & 0 & 0 & 0 \\
0 & 0 & 0 & \frac{1}{2} & 0 & 0 \\
0 & 0 & 0 & 0 & \frac{1}{2} & \\
0 & 0 & 0 & 0 & 0 & \frac{1}{2}
\end{array}\right]
$$

with

$$
\begin{aligned}
& \mathrm{K}_{\mathrm{V}}=\frac{1}{9} \mathrm{C}_{\text {ppqq }} \\
& \mathrm{G}_{\mathrm{V}}=\frac{1}{10}\left(\mathrm{C}_{\text {pqpq }}-\frac{1}{3} \mathrm{C}_{\text {ppqq }}\right)
\end{aligned}
$$

$K_{v}$ and $G_{v}$ are the well known Voigt average polycrystalline bulk and shear modulus, respectively. Hence, the symmetric fourth rank elastic tensor of isotropic media is decomposed into two orthogonal terms. Equation (23) indicates that the isotropic symmetric fourth rank tensor, $\mathrm{C}_{\mathrm{ijkm}}$, is a subset of the general symmetric fourth rank elastic tensor, and decomposed into two terms, each of which has a distinct physical meaning, and the two terms are the same terms consisting the isotropic case. It is easily verified that the decomposed tensors form an orthogonal set, and their sum is the isotropic symmetric fourth rank tensor, $C_{i j k m}$, which is identical to those found by Nye (Nye, 1959).

In fact, the above results are the same as those given in (Tu, 1968; Nye, 1959; Voigt, 1889; Radwan, 1991; Ikeda, 1990). Thus, it has been established that macroscopically isotropic elastic coefficients, which were obtained by W. Voigt, can be obtained directly from the procedure developed. Moreover, this procedure is valid for the most anisotropic triclinic elastic tensor.

\section{The concept of norm}

The comparison of magnitudes of the norms can give valuable information about the origin of the physical property under examination. Since the norm is invariant in the material, the norm of a Cartesian tensor may be used as the most suitable representing and comparing the overall effect of a certain property of anisotropic materials of the same or different symmetry or the same material with different phases based on the crystallographic level (Spencer, 1983; Srinivasan, 1998; Tu, 1968; Nye, 1959; Voigt, 1889; Radwan, 1991; Ikeda, 1990). The larger the norm value, the more effective the property is. Generalizing the 
concept of the modulus of a vector, a norm of a Cartesian tensor is defined as the square root of the contracted product over all the indices with itself (Srinivasan, 1998; Tu, 1968; Radwan, 1991). Since the constructed basis in this method is orthonormal and $C_{i j k m}$ is in the space spanned by that orthonormal basis, the norm for the elastic stiffness, for example, is given by:

$$
N=\|C\|=\left\{C_{i j} \cdot C_{i j}\right\}^{1 / 2}
$$

\subsection{A proposed relation between the norm ratio and the anisotropy degree}

It is known that the anisotropy of the material, i.e., the symmetry group of the material and the anisotropy of the measured property depicted in the same material may be quite different. Obviously, the property tensor must show, at least, the symmetry of the material. For example, a property which is measured in a material can almost be isotropic but the material symmetry group itself may have very few symmetry elements.

In the elastic stiffness tensors, the isotropic symmetry material is decomposed into two parts, the decomposition of the cubic symmetry material is consisted of the same two isotropic decomposed parts and a third part, and the decomposition of the hexagonal symmetry material is consisted of the same two isotropic decomposed parts and another three parts. Consequently, the Norm Ratio Criteria (NRC) proposed in this chapter is close to that proposed in (Gaith \& Alhayek, 2009; Gaith \& Akgoz, 2005). For isotropic materials, the elastic stiffness tensor has two parts, so the norm of the elastic stiffness tensor for isotropic materials is equal to the norm of these two parts, i.e., $\mathrm{N}=\mathrm{N}_{\text {iso }}$. Hence, the ratio $\left(\frac{\mathrm{N}_{\text {iso }}}{\mathrm{N}}=1\right)$ for isotropic materials. For cubic symmetry materials the elastic stiffness tensor has the same two parts that consisting the isotropic symmetry materials and a third, will be designated as the other than isotropic or the anisotropic part, so two ratios are defined: $\frac{\mathrm{N}_{\text {iso }}}{\mathrm{N}}$ for the isotropic parts and $\frac{\mathrm{N}_{\text {anis }}}{\mathrm{N}}$ for the anisotropic part. For more anisotropic materials, the elastic stiffness tensor additionally contains more anisotropic parts, so $\frac{\mathrm{N}_{\text {anis }}}{\mathrm{N}}$ is defined for all the anisotropic parts.

Although the norm ratios of different parts represent the anisotropy of that particular part, they can also be used to asses the anisotropy degree of a material property as a whole, in this chapter the following criteria are proposed:

1. When $\mathrm{N}_{\text {iso }}$ is dominating among norms of the decomposed parts, the closer the norm ratio $\frac{\mathrm{N}_{\text {iso }}}{\mathrm{N}}$ is to one, the closer the material property is isotropic.

2. When $\mathrm{N}_{\text {iso }}$ is not dominating or not present, norms of the other parts can be used as a criterion. But in this case the situation is reverse; the larger the norm ratio value, the more anisotropic the material property is. 


\section{Applications}

\subsection{Piezoelectric semiconductors and ceramics}

Among semiconductor crystals, a family of wurtzite- type belongs to the $6 \mathrm{~mm}$ class, which is piezoelectric active. The material properties (Tsai, 1992) and the norm calculations are in Table 1. From the table, the most piezoelectric effective among the five materials is CdS which has a very important feature in the thin films of semiconductors. For piezoelectric ceramics, the most potential piezoelectric material because of its higher strength, high rigidity and more importantly the better piezoelectricity, Table 2 includes the piezoelectric coefficients (Temple, 1960) and calculated norms. From the table, the most effective piezoelectric among the seven ceramics is PZT-5H.

\begin{tabular}{|l|c|c|c|c|}
\hline Material & $\mathrm{d}_{11}$ & $\mathrm{~d}_{33}$ & $\mathrm{~d}_{15}$ & $\mathrm{~N}$ \\
\hline $\mathrm{BeO}$ & -0.12 & 0.24 & & 0.29 \\
\hline $\mathrm{ZnO}$ & -5.0 & 12.4 & -8.3 & 18.48 \\
\hline $\mathrm{CdS}$ & -5.2 & 10.3 & -14.0 & 23.50 \\
\hline CdSe & -3.9 & 7.8 & -10.0 & 17.07 \\
\hline
\end{tabular}

Table 1. The Constants and Norms of Piezoelectric Semiconductors[10-12 C N-1]

\begin{tabular}{|l|c|c|c|c|}
\hline Material & $\mathrm{d}_{11}$ & $\mathrm{~d}_{33}$ & $\mathrm{~d}_{15}$ & $\mathrm{~N}$ \\
\hline PZT-4 & -5.2 & 15.1 & 12.7 & 24.59 \\
\hline PZT-5 & -5.4 & 15.8 & 12.3 & 24.71 \\
\hline BaTi O3 & -4.35 & 17.5 & 11.4 & 18.82 \\
\hline PZT-5H & -6.5 & 23.3 & 17 & 34.72 \\
\hline PZT-6B & -0.9 & 7.1 & 4.6 & 9.71 \\
\hline PZT-8 & -4.0 & 23.3 & 10.4 & 28.13 \\
\hline C-24 & 1.51 & 8.53 & 3.89 & 10.37 \\
\hline
\end{tabular}

Table 2. The Constants and Norms of Piezoelectric Ceramics $\left[10^{-12} \mathrm{C} \mathrm{N}^{-1}\right]$

\subsection{Fiber reinforced composite materials}

Under specific couplings of the elastic constants of orthotropic media, a very important family of orthotropic materials degenerates into the class of either transversely isotropic or isotropic media. Most of the engineering composites, especially fiber-reinforced, are of transversely isotropic media. Hence, for different composites, the norms are calculated for each material (Radwan, 1991) in Table 3. From the table, it can be clearly concluded that B(4)/N5505 has the strongest stiffness effect among the five composites. From Table 4, the most isotropic composite is E-glass/epoxy, and the most anisotropic composite is T300/5208. From the latter table, it was possible to measure the degree of anisotropy for several composites. 


\begin{tabular}{|l|c|c|c|c|c|c|c|}
\hline Materials & $\mathrm{C}_{11}$ & $\mathrm{C}_{22}$ & $\mathrm{C}_{12}$ & $\mathrm{C}_{23}$ & $\mathrm{C}_{44}$ & $\mathrm{C}_{55}$ & Norm \\
\hline T300/5208 & 184.60 & 13.94 & 5.88 & 7.06 & 3.44 & 7.17 & 174.06 \\
\hline $\mathrm{B}(4) / \mathrm{N} 5505$ & 208.08 & 25.04 & 95.72 & 12.70 & 6.17 & 5.59 & 284.62 \\
\hline AS/H3501 & 141.80 & 12.20 & 85.08 & 6.21 & 3.00 & 7.10 & 222.11 \\
\hline E-lass/Epoxy & 41.12 & 11.57 & 21.38 & 6.04 & 2.77 & 4.14 & 62.58 \\
\hline Kev 9/Epoxy & 78.66 & 7.53 & 53.49 & 3.86 & 1.83 & 2.30 & 132.92 \\
\hline
\end{tabular}

Table 3. Elastic constants and norms for transversely isotropic materials, GPa

\begin{tabular}{|l|c|c|c|c|c|}
\hline Material & $\mathrm{N}_{\text {iso }}$ & $\mathrm{N}_{\text {aniso }}$ & $\mathrm{N}$ & $\frac{\mathrm{N}_{\text {iso }}}{\mathrm{N}}$ & $\frac{\mathrm{N}_{\text {anis }}}{\mathrm{N}}$ \\
\hline $\mathrm{T} 300 / 5208$ & 99.67 & 142.64 & 174.06 & 0.56 & 0.82 \\
\hline $\mathrm{B}(4) / \mathrm{N} 5505$ & 223.39 & 176.37 & 284.62 & 0.78 & 0.62 \\
\hline AS/H3501 & 173.04 & 139.24 & 222.11 & 0.78 & 0.63 \\
\hline E-glass/Epoxy & 55.01 & 29.84 & 62.58 & 0.88 & 0.48 \\
\hline Kev 49/Epoxy & 105.13 & 81.34 & 132.92 & 0.79 & 0.61 \\
\hline
\end{tabular}

Table 4. The Norm ratios for transversely isotropic materials, GPa

\subsection{II-IV semiconductor compounds $\mathrm{ZnX}(\mathrm{X}=\mathrm{S}$, Se, Te)}

Covalent materials such as II-IV semiconductor compounds $\mathrm{ZnX}(X=S, \mathrm{Se}, \mathrm{Te})$ have been extensively studied for their intrinsic structural, optical, and elastic properties such as energy gap, charge density, lattice constants and bulk modulus. However, bulk modulus has been found to correlate well with strength and hardness in many materials and those with largest bulk moduli are usually expected to be the hardest materials. Therefore, one of the important parameters that characterize the physical property of a material system is the material stiffness and its corresponding bulk modulus which measures the degree of stiffness or the energy required to produce a given volume deformation. The bulk modulus reflects important bonding characters in the material and, for many applications, is used as an indicator for material strength and hardness. Early experimental and theoretical investigations for bulk modulus were reported in (Cohen, 1985; Lam et al., 1987). Cohen (Cohen, 1985) obtained an empirical expression for the bulk modulus based on the nearest-neighbour distance. His theoretical and experimental results were in agreement. Lam et al. (Lam, 1987) obtained an expression for bulk modulus using the total energy method with acceptable results. The bulk modulus for the semiconductor compounds was found to be inverse proportionally correlated to the lattice constants (Lam et al., 1987; Al-Douri et al., 2004).

Physical properties are intrinsic characteristics of matter that are not affected by any change of the coordinate system. Therefore, tensors are necessary to define the intrinsic properties of the medium that relate an intensive quantity (i.e. an externally applied stimulus) to an extensive thermodynamically conjugated one (i.e. the response of the medium). Such intrinsic properties are the dielectric susceptibility, piezoelectric, and the elasticity tensors. An interesting feature of the decompositions is that it simply and fully takes into account the symmetry properties when relating macroscopic effects to microscopic phenomena. One can directly show the influence of the crystal structure on physical properties, for instance, 
when discussing macroscopic properties in terms of the sum of the contributions from microscopic building units (chemical bond, coordination polyhedron, etc). A significant advantage of such decompositions is to give a direct display of the bearings of the crystal structure on the physical property.

The proposed procedure in this chapter has introduced a method to measure the stiffness and piezoelectricity in fiber reinforced composite and piezoelectric materials using the norm criterion on the crystal scale. In this method, norm ratios proposed to measure the degree of anisotropy in an anisotropic material, and compare it with other materials of different symmetries. It was able to segregate the anisotropic material property into two parts: isotropic and anisotropic parts. Of the new insights provided by invariance considerations, the most important is providing a complete comparison of the magnitude of a given property in different crystals. Such a comparison could be obvious for average refractive index, even birefringence, piezoelectricity, electro-optic effects, elasticity, etc. From a device point of view, the new insights facilitate the comparison of materials; one is interested in maximizing the fig. of merit by choosing the optimum configuration (crystal cut, wave propagation direction and polarization, etc); and one wants to be able to state that a particular material is better than another for making a transducer or modulator (Jerphagnon, 1978). It is most suitable for a complete quantitative comparison of the strength or the magnitude of any property in different materials belonging to the same crystal class, or different phases of the same material. The norm concept is very effective for selecting suitable materials for electro-optic devices, transducers, modulators, acousto-optic devices. Therefore, using the elastic constant for anisotropic material, an elastic stiffness scale for such anisotropic material, and a scale to measure the isotropic elasticity within the material will be discussed. Besides, the microscopic origin of the overall elastic stiffness and bulk modulus calculation will be correlated with the structural properties parameter, i.e. lattice constant a, which represents some fundamental length scale for the chemical bond of the unit cell.

The elastic stiffness matrix representation for the isotropic system can be decomposed in a contracted form as:

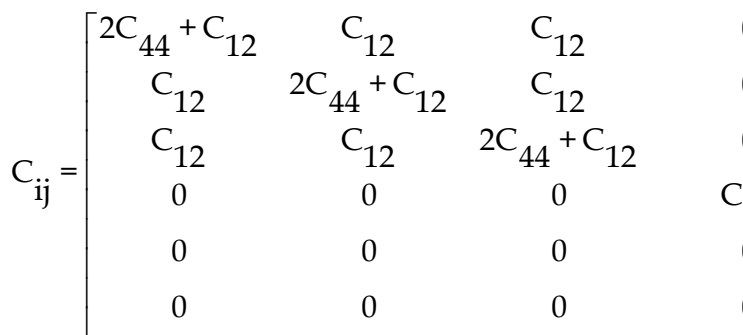

$\left.\begin{array}{ccc}0 & 0 & 0 \\ 0 & 0 & 0 \\ 0 & 0 & 0 \\ \mathrm{C}_{44} & 0 & 0 \\ 0 & \mathrm{C}_{44} & 0 \\ 0 & 0 & \mathrm{C}_{44}\end{array}\right]$

$$
=A_{1}\left[\begin{array}{llllll}
1 & 1 & 1 & 0 & 0 & 0 \\
1 & 1 & 1 & 0 & 0 & 0 \\
1 & 1 & 1 & 0 & 0 & 0 \\
0 & 0 & 0 & 0 & 0 & 0 \\
0 & 0 & 0 & 0 & 0 & 0 \\
0 & 0 & 0 & 0 & 0 & 0
\end{array}\right]+A_{2}\left[\begin{array}{cccccc}
4 & -2 & -2 & 0 & 0 & 0 \\
-2 & 4 & -2 & 0 & 0 & 0 \\
-2 & -2 & 4 & 0 & 0 & 0 \\
0 & 0 & 0 & 3 & 0 & 0 \\
0 & 0 & 0 & 0 & 3 & 0 \\
0 & 0 & 0 & 0 & 0 & 3
\end{array}\right]
$$


where

$$
\begin{aligned}
& A_{1}=\frac{1}{3}\left(C_{11}+2 C_{12}\right), C_{11}=2 C_{44}+C_{12} \\
& A_{2}=\frac{1}{15}\left(C_{11}-C_{12}+3 C_{44}\right)
\end{aligned}
$$

where $\mathrm{A}_{1}$ and $\mathrm{A}_{2}$ are the Voigt average polycrystalline bulk $\mathrm{B}$ and shear $\mathrm{G}$ modulus, respectively. The decomposed parts of Eq. (25) designated as bulk and shear modulus are identical to those found in literature (Voigt, 1889; Hearmon, 1961; Pantea et al., 2009).

For cubic crystals such as the II-VI semiconductor compounds, there are only three independent elastic stiffness coefficients $C_{11}, C_{12}$, and $C_{44}$ that can describe the mechanical elastic stiffness for these materials. The elastic coefficient $C_{11}$ is the measure of resistance to deformation by a stress applied on the (100) plane, while $C_{44}$, represents the measure of resistance to deformation with respect to a shearing stress applied across the (100) plane. These elastic coefficients are function of elastic material parameters, namely, Young's modulus, shear modulus, and Poisson's ratio. Thus, using the orthonormal decomposition procedure (Gaith \& Akgox, 2005), the elastic stiffness matrix representation for cubic system can be decomposed in a contracted form as:

$$
\begin{aligned}
& \mathrm{C}_{\mathrm{ij}}=\left[\begin{array}{cccccc}
\mathrm{C}_{11} & \mathrm{C}_{12} & \mathrm{C}_{12} & 0 & 0 & 0 \\
\mathrm{C}_{12} & \mathrm{C}_{11} & \mathrm{C}_{12} & 0 & 0 & 0 \\
\mathrm{C}_{12} & \mathrm{C}_{12} & \mathrm{C}_{11} & 0 & 0 & 0 \\
0 & 0 & 0 & \mathrm{C}_{44} & 0 & 0 \\
0 & 0 & 0 & 0 & \mathrm{C}_{44} & 0 \\
0 & 0 & 0 & 0 & 0 & \mathrm{C}_{44}
\end{array}\right]=\mathrm{A}_{1}\left[\begin{array}{cccccc}
1 & 1 & 1 & 0 & 0 & 0 \\
1 & 1 & 1 & 0 & 0 & 0 \\
1 & 1 & 1 & 0 & 0 & 0 \\
0 & 0 & 0 & 0 & 0 & 0 \\
0 & 0 & 0 & 0 & 0 & 0 \\
0 & 0 & 0 & 0 & 0 & 0
\end{array}\right] \\
& +A_{2}\left[\begin{array}{cccccc}
4 & -2 & -2 & 0 & 0 & 0 \\
-2 & 4 & -2 & 0 & 0 & 0 \\
-2 & -2 & 4 & 0 & 0 & 0 \\
0 & 0 & 0 & 3 & 0 & 0 \\
0 & 0 & 0 & 0 & 3 & 0 \\
0 & 0 & 0 & 0 & 0 & 3
\end{array}\right]+\mathrm{A}_{3}\left[\begin{array}{ccccccc}
-4 & 2 & 2 & 0 & 0 & 0 \\
2 & 4 & -2 & 0 & 0 & 0 \\
2 & 2 & -4 & 0 & 0 & 0 \\
0 & 0 & 0 & 2 & 0 & 0 \\
0 & 0 & 0 & 0 & 2 & 0 \\
0 & 0 & 0 & 0 & 0 & 2
\end{array}\right]
\end{aligned}
$$

where

$$
\begin{aligned}
& A_{1}=\frac{1}{3}\left(C_{11}+2 C_{12}\right) \\
& A_{2}=\frac{1}{15}\left(C_{11}-C_{12}+3 C_{44}\right) \\
& A_{3}=\frac{1}{10}\left(C_{12}-C_{11}+2 C_{44}\right)
\end{aligned}
$$


It can be shown that the sum of the three orthonormal parts on the right hand side of Eq. (27) is apparently the main matrix of cubic system (Hearmon, 1961). Also, the first two terms on the right hand side are identical to the corresponding two terms obtained in Eq. (23) for the isotropic system (Hearmon, 1961). Hence, it can be stated that the cubic system is discriminated into the sum of two parts: isotropic part (first two terms), and anisotropic part (third term). The latter term resembles the contribution of the anisotropy on elastic stiffness in the cubic system. On the other hand, the first term on the right hand side of Eqs (23) and (27), designated as the bulk modulus, is identical to Voigt bulk modulus (Hearmon, 1961).

Nowadays, the necessity of alternative energy use is widely accepted. In solar energy technology, thin film solar technology based on the II-IV semiconductor compounds, is very promising due to lower production costs and shorter energy pay back times (Fischer, 2006). For these compounds, a successful interface between absorber and buffer layers with alternative and promising non-toxic materials requires compositional and electronic material characterization as a prerequisite for understanding and intentionally generating interfaces in photovoltaic devices (Fischer, 2006). On the other hand, stability of $\mathrm{ZnTe} / \mathrm{ZnS}$ solar cells is of concern for their application in space, where the cells have to withstand high energy particles, mainly electrons and protons that can cause severe damage in solar cells up to a complete failure. Therefore, the radiation hardness and damage mechanism of the ZnTe solar cells is associated with the overall elastic stiffness and bulk modulus (Bätzner, 2004). Table 5 presents the materials elastic stiffness coefficients, calculated bulk $\mathrm{B}$ and overall elastic stiffness $\mathrm{N}$ moduli for the II-VI semiconductor $\mathrm{ZnX}(\mathrm{X}=\mathrm{S}$, Se, and Te) compounds. Fig. 1 shows clearly the correlation between overall elastic stiffness $N$ and bulk modulus B. Quantitatively, the overall elastic stiffness increases as the calculated bulk modulus B increases. Besides, the calculated bulk moduli are identical to those found by theory of anisotropy (Hearmon, 1961), and are in agreement with experimental values (Cohen, 1985) with maximum error of $8.5 \%$ for ZnTe. The calculated bulk moduli obey the cubic stability conditions, meaning that $\mathrm{C}_{12} \leq \mathrm{B} \leq \mathrm{C}_{11}$. Fig. 2 shows that the bulk modulus is inversely proportional to lattice constants a which was confirmed in several studies (Lam et al., 1987; Al-Douri et al., 2004). Consequently, from Fig. 3 the overall elastic stiffness $\mathrm{N}$ is inversely proportional to lattice constants a, as well. Fig. 1-3 indicate that among the three compounds under examination, ZnS has the largest elastic stiffness, largest bulk modulus (lowest compressibility), and lowest lattice constant, while ZnTe, in contrary, has the smallest elastic stiffness, smallest bulk modulus, and largest lattice constant. Therefore, the overall elastic stiffness and bulk modulus, the only elastic moduli possessed by all states of matter, reveal much about interatomic bonding strength. The bulk modulus also is the most often cited elastic constant to compare interatomic bonding strength among various materials (Pantea, 2009), and thereafter the overall elastic stiffness can be cited as well.

For the isotropic symmetry material, the elastic stiffness tensor is decomposed into two parts as shown in Eq. (23), meanwhile, the decomposition of the cubic symmetry material, from Eq. (27), is consisted of the same two isotropic decomposed parts and a third part. It can be verified the validity of this trend for higher anisotropy, i.e., any anisotropic 


\begin{tabular}{|c|c|c|c|c|c|c|c|}
\hline & C11 & C12, & C44 & N & B & $\begin{array}{c}\text { Bexp } \\
\text { (Cohen, } \\
1985)\end{array}$ & $\begin{array}{c}\text { a } \\
\text { (Chelikowsky, } \\
1987)\end{array}$ \\
\hline ZnS & 104 & 65 & 46.2 & 266.5 & 78 & 77 & 0.54 \\
\hline ZnSe & 95.9 & 53.6 & 48.9 & 244.0 & 67.7 & 64.7 & 0.57 \\
\hline ZnTe & 82 & 42 & 55 & 224.0 & 55.3 & 51 & 0.61 \\
\hline
\end{tabular}

Table 5. Elastic coefficients (GPa) (Cohen, 1985), overall stiffness N (GPa), bulk modulus B (GPa), and lattice constants a (nm)

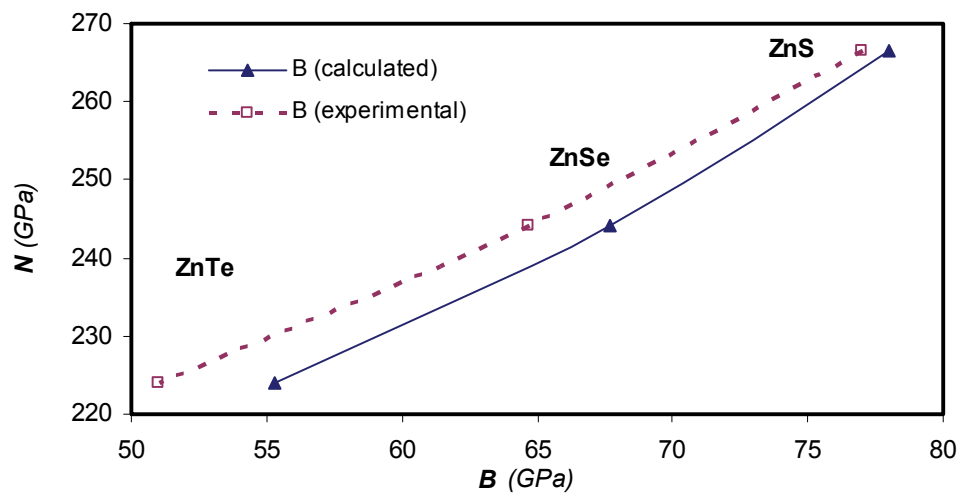

Fig. 1. The overall elastic stiffness $N$ versus bulk modulus B for $\operatorname{ZnX}(X=S, S e, T e)$

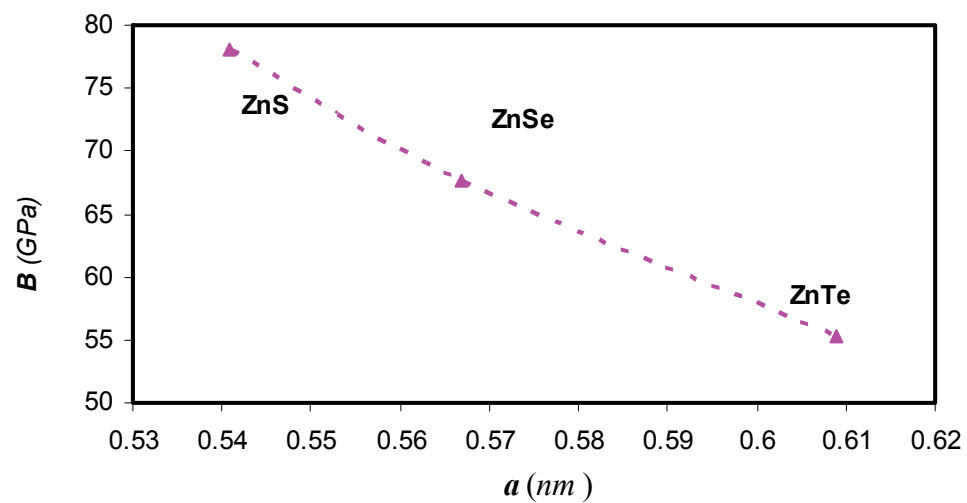

Fig. 2.The bulk modulus $B$ versus the lattice constants a for $Z n X(X=S$, Se, Te) 


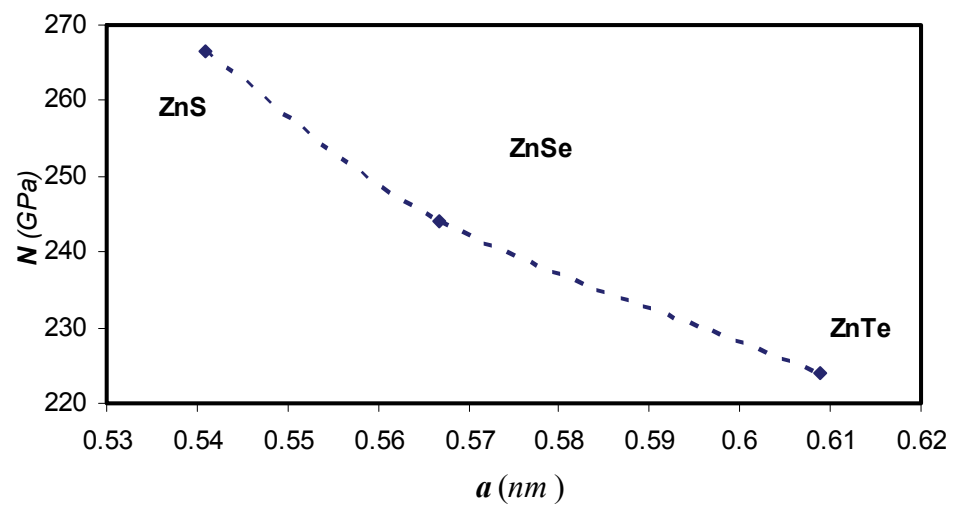

Fig. 3. The overall stiffness $N$ versus the lattice constants a for $Z n X(X=S, S e, T e)$

\begin{tabular}{|l|c|c|c|c|c|c|c|}
\hline & Niso & Naniso & $\mathrm{N}$ & $\frac{\mathrm{N}_{\text {iso }}}{\mathrm{N}}$ & $\frac{\mathrm{N}_{\text {anis }}}{\mathrm{N}}$ & $\mathrm{B}$ & $\mathrm{A}$ \\
\hline $\mathrm{ZnS}$ & 262 & 49 & 266.5 & 0.9830 & 0.1836 & 78 & 0.54 \\
\hline ZnSe & 238.7 & 50.9 & 244.0 & 0.9780 & 0.2084 & 67.7 & 0.57 \\
\hline ZnTe & 214.6 & 64.2 & 224.0 & 0.9581 & 0.2865 & 55.3 & 0.61 \\
\hline
\end{tabular}

Table 6. The overall elastic stiffness N (GPa) and norm ratios for the II-IV semiconductor compounds $\mathrm{ZnS}$, ZnSe, and ZnTe

The norms and norm ratios for ZnS, ZnSe, and ZnTe are calculated and presented in Table 6. From the table, interesting phenomena are observed; as the isotropic ratio $\frac{\mathrm{N}_{\text {iso }}}{\mathrm{N}}$ increases, the anisotropic ratio $\frac{\mathrm{N}_{\text {anis }}}{\mathrm{N}}$ decreases, which confirms the definitions of these two ratios, and the bulk modulus increases at the same time. Therefore, the nearest material to isotropy (or least anisotropy) is $\mathrm{ZnS}$, with $\frac{\mathrm{N}_{\text {iso }}}{\mathrm{N}}=0.9830$, in which corresponds to the largest bulk modulus B $=78 \mathrm{GPa}$. The least isotropic (or nearest to anisotropy) is ZnTe, with $\frac{\mathrm{N}_{\text {iso }}}{\mathrm{N}}=$ 0.9581, in which corresponds to the smallest bulk modulus B $=55.3 \mathrm{GPa}$. Since the cubic system is the nearest to isotropy among the anisotropic systems, the calculated values of isotropic norm ratio, $\frac{N_{\text {iso }}}{N}$, in Table 2 are very close to one (i.e., $0.9581-0.9830$ ). Hence, it can be concluded that the closer the material is to isotropy, the larger the bulk modulus for 
the material is. A reverse trend correlating the norm ratios and lattice constants can be seen from the table; the closer the material is to isotropy, the smaller the lattice constant for the material is.

The overall elastic stiffness of II-IV semiconductor compounds ZnS, ZnSe, and ZnTe is calculated and found to be directly proportional to bulk modulus and inversely proportional to lattice constants. Among these compounds, ZnS has the largest overall elastic stiffness and bulk modulus and the smallest lattice constant. Meanwhile, ZnTe has the smallest overall elastic stiffness and bulk modulus and the largest lattice constant. The Norm Ratio Criteria (NRC) is introduced to scale and measure the isotropy in the cubic system material among the semiconductor compounds ZnS, ZnSe, and ZnTe. Hence, a scale quantitative comparison of the contribution of the anisotropy to the elastic stiffness and to measure the degree of anisotropy in an anisotropic material is proposed. $\mathrm{ZnS}$ is the nearest to isotropy (or least anisotropic) while $\mathrm{ZnTe}$ is the least isotropic (or nearest to anisotropic) among these compounds. These conclusions can be investigated on the II-IV semiconductor compounds $\mathrm{CdX}(\mathrm{X}=\mathrm{S}, \mathrm{Se}, \mathrm{Te})$ in similar manner.

\subsection{The hetrojunction layers compounds in solar photovoltaic cells: CulnSe2, CulnS2, AgGaSe2, and AgGaS2}

Various attempts have been made to correlate the bulk modulus of compound semiconductors and chalcopyrite compounds with many other physical parameters. Nevertheless, it is found that bulk modulus interconnected well with strength and hardness in many materials (Choi \& Yu, 1996). Therefore, the material stiffness and its corresponding bulk modulus is one of the important factors that characterize the physical property of a material system which quantifies the degree of stiffness or the energy required to produce a given volume deformation. With a good agreement result, an empirical expression for the bulk modulus was obtained by Cohen (Cohen, 1985) based on the nearest-neighbour distance. Using the total energy method Lam et al. (Lam et al., 1987) obtained an expression for bulk modulus with acceptable results. The bulk modulus for the semiconductor compounds was found to be inverse proportionally correlated to the lattice constants (Lam et al., 1987 \& Al-Douri, 2004). Gaith et al (Gaith \& Alhayek, 2009) have studied the correlation between the bulk modulus and the over all stiffness and lattice constants for CdX and $\mathrm{ZnX}(\mathrm{X}=\mathrm{S}$, Se, and Te) using orthonormal decomposition method (ODM) (Gaith \& Alhayek, 2009) from continuum mechanics point of view.

The purpose of this section is to understand how qualitative ground state concepts of the hetrojunction layer compounds, CuInSe2, CuInS2, AgGaSe2, and AgGaS2, such as overall elastic stiffness, can be related to bulk modulus and lattice constants. Therefore, using the elastic coefficients for anisotropic material, an elastic stiffness scale for such anisotropic material, and a scale to quantify the isotropic elasticity within the material will be discussed. The number of elements of the decomposed stiffness tensor should be equal to the number of non-vanishing distinct stiffness coefficients, i.e., six constants for tetragonal materials, that can completely describe the elastic stiffness in that medium. Thus, using Orthonormal Decomposition Method (ODM), namely, Orthonormal Tensor Basis Method (OTBM) (Gaith \& Alhayek, 2009; Gaith \& Akgoz, 2005), the matrix representation for the elastic stiffness with tetragonal symmetry system is decomposed into a contracted form as shown in Eq. (29): 


$$
\begin{aligned}
& \mathrm{C}_{\mathrm{ij}}=\left[\begin{array}{cccccc}
\mathrm{C}_{11} & \mathrm{C}_{12} & \mathrm{C}_{13} & 0 & 0 & 0 \\
\mathrm{C}_{12} & \mathrm{C}_{11} & \mathrm{C}_{13} & 0 & 0 & 0 \\
\mathrm{C}_{13} & \mathrm{C}_{13} & \mathrm{C}_{33} & 0 & 0 & 0 \\
0 & 0 & 0 & \mathrm{C}_{44} & 0 & 0 \\
0 & 0 & 0 & 0 & \mathrm{C}_{44} & 0 \\
0 & 0 & 0 & 0 & 0 & \mathrm{C}_{66}
\end{array}\right]=\mathrm{A}_{1}\left[\begin{array}{cccccc}
1 & 1 & 1 & 0 & 0 & 0 \\
1 & 1 & 1 & 0 & 0 & 0 \\
1 & 1 & 1 & 0 & 0 & 0 \\
0 & 0 & 0 & 0 & 0 & 0 \\
0 & 0 & 0 & 0 & 0 & 0 \\
0 & 0 & 0 & 0 & 0 & 0
\end{array}\right]+ \\
& +A_{2}\left[\begin{array}{cccccc}
4 & -2 & -2 & 0 & 0 & 0 \\
-2 & 4 & -2 & 0 & 0 & 0 \\
-2 & -2 & 4 & 0 & 0 & 0 \\
0 & 0 & 0 & 3 & 0 & 0 \\
0 & 0 & 0 & 0 & 3 & 0 \\
0 & 0 & 0 & 0 & 0 & 3
\end{array}\right]+A_{3}\left[\begin{array}{cccccc}
-3 & -1 & -1 & 0 & 0 & 0 \\
-1 & -3 & -1 & 0 & 0 & 0 \\
-1 & -1 & 12 & 0 & 0 & 0 \\
0 & 0 & 0 & -1 & 0 & 0 \\
0 & 0 & 0 & 0 & -1 & 0 \\
0 & 0 & 0 & 0 & 0 & -1
\end{array}\right]+ \\
& +A_{4}\left[\begin{array}{cccccc}
-3 & -5 & 4 & 0 & 0 & 0 \\
-5 & -3 & 4 & 0 & 0 & 0 \\
4 & 4 & 0 & 0 & 0 & 0 \\
0 & 0 & 0 & 1 & 0 & 0 \\
0 & 0 & 0 & 0 & 1 & 0 \\
0 & 0 & 0 & 0 & 0 & 1
\end{array}\right]+A_{5}\left[\begin{array}{cccccc}
-1 & 1 & 0 & 0 & 0 & 0 \\
1 & -1 & 0 & 0 & 0 & 0 \\
0 & 0 & 0 & 0 & 0 & 0 \\
0 & 0 & 0 & 1 & 0 & 0 \\
0 & 0 & 0 & 0 & 1 & 0 \\
0 & 0 & 0 & 0 & 0 & -1
\end{array}\right]+ \\
& +\mathrm{A}_{6}\left[\begin{array}{cccccc}
-1 & 1 & 0 & 0 & 0 & 0 \\
1 & -1 & 0 & 0 & 0 & 0 \\
0 & 0 & 0 & 0 & 0 & 0 \\
0 & 0 & 0 & 0 & 0 & 0 \\
0 & 0 & 0 & 0 & 0 & 0 \\
0 & 0 & 0 & 0 & 0 & 1
\end{array}\right]
\end{aligned}
$$

where

$$
\begin{aligned}
& A_{1}=\frac{1}{90}\left(2 C_{11}+2 C_{12}+C_{33}+4 C_{13}\right) \\
& A_{2}=\frac{1}{45}\left(2 C_{11}-C_{12}+6 C_{44}+3 C_{66}+C_{33}-2 C_{13}\right) \\
& A_{3}=\frac{1}{90}\left(-3 C_{11}-C_{12}-4 C_{44}-2 C_{66}+6 C_{33}-2 C_{13}\right) \\
& A_{4}=\frac{1}{144}\left(-6 C_{11}-10 C_{12}+8 C_{44}+4 C_{66}+16 C_{13}\right) \\
& A_{5}=\frac{1}{16}\left(-2 C_{11}+2 C_{12}+8 C_{44}-4 C_{66}\right) \\
& A_{6}=\frac{1}{8}\left(-2 C_{11}+2 C_{12}+4 C_{66}\right)
\end{aligned}
$$


Where $\mathrm{A}_{1}$ to $\mathrm{A}_{6}$ are constants in terms of elastic stiffness coefficients expressed as in Eq.

(30). It can be observed clearly that the first two terms on the right hand side are identical to the corresponding well known two terms namely bulk and shear (Gaith \& Akgoz, 2005) which are identical to those found in literature (Voigt, 1889) for the isotropic system (Hearmon, 1961). Here, $A_{1}$ and $A_{2}$ defined in Eq. (2), are the Voigt average polycrystalline

bulk B and shear modulus G, respectively. Hence, it can be stated that the tetragonal system is discriminated into the sum of two parts: isotropic part (first two terms), and anisotropic part (other four terms). The latter part resembles the contribution of the anisotropy on elastic stiffness in the tetragonal system.

In solar energy technology, thin film solar technology based on the hetrojunction layer compounds CuInSe2, CuInS2, AgGaSe2, and AgGaS2, is very promising due to lower production costs and shorter energy pay back times (Fischeret al., 2006). For these compounds, the successful interface between absorber and buffer layers with alternative materials requires structural and optical material characterization as a prerequisite for understanding interfaces in photovoltaic devices (Fischeret al., 2006). On the other hand, stability of these compounds in solar cells is of concern due to their application in space, where the cells have to withstand high energy particles, mainly electrons and protons that can cause severe damage in solar cells up to a complete failure. Therefore, the radiation hardness and damage mechanism of the hetrojunction layer compounds solar cells is associated with the overall elastic stiffness and bulk modulus (Bätzner et al., 2004). The materials elastic stiffness coefficients and lattice constants for CuInSe2, CuInS2, AgGaSe2, and AgGaS2 are presented in Table 7. The correlation trend between overall elastic stiffness $\mathrm{N}$ and bulk modulus B for each group, i.e. (CuInS2, CuInSe2) and (AgGaS2, AgGaSe2) is clearly shown in Fig. 4; the overall elastic stiffness increases as the calculated bulk modulus B increases. Besides, the calculated bulk moduli are in good agreement with those found by theory of anisotropy (Hearmon, 1961) and experimental values (Cohen, 1985). Also, the bulk modulus for each group is inversely proportional to lattice constants a, as shown in Fig. 5, which was confirmed in several studies (Lam et al., 1987; Al-Douri et al., 2004; (Christensen \& Christensen, 1986; Al-Douri et al., 2001). Consequently, the overall elastic stiffness $\mathrm{N}$ is inversely proportional to lattice constants a, as shown in fig. 3. CuInS2 and AgGaS2 have larger elastic stiffness, largest bulk modulus, and lower lattice constant than those for CuInSe2 and AgGaSe2, respectively. Therefore, the overall elastic stiffness and bulk modulus, the only elastic moduli possessed by all states of matter, reveal much about interatomic bonding strength. The bulk modulus also is the most often cited elastic constant to compare interatomic bonding strength among various materials (Pantea et al., 2009), and thereafter the overall elastic stiffness can be cited as well (Gaith \& Alhayek, 2009).

For the isotropic symmetry material, the elastic stiffness tensor is decomposed into two parts (Kim \& Chen, 2004; Spencer, 1983; Voigt, 1889; Hearmon, 1961); meanwhile, the decomposition of the tetragonal symmetry material, from Eq. (29) is consisted of the same two isotropic decomposed parts and other four terms. The Norm Ratio Criteria (NRC) used in here is similar to that proposed in (Gaith \& Alhayek, 2009; Gaith \& Akgoz, 2005). For tetragonal symmetry materials, the elastic stiffness tensor has the same two parts that consisting the isotropic symmetry materials and the other four terms, will be designated as 
the other than isotropic or the anisotropic part. Hence, two ratios are defined as: $\frac{\mathrm{N}_{\text {iso }}}{\mathrm{N}}$ for the isotropic parts and $\frac{\mathrm{N}_{\text {anis }}}{\mathrm{N}}$ for the anisotropic parts. The norm ratios can also be used to assess the degree of anisotropy of a material property as a whole. The norms and norm ratios for the hetrojunction layer compounds CuInSe2, CuInS2, AgGaSe2, and AgGaS2 are calculated and shown in Fig. 7 and 8; as the isotropic ratio $\frac{\mathrm{N}_{\text {iso }}}{\mathrm{N}}$ increases, the anisotropic ratio $\frac{\mathrm{N}_{\text {anis }}}{\mathrm{N}}$ decreases and this confirms the definitions of these two ratios, and the bulk modulus increases at the same time.

Therefore, CuInS2 is a closer material to isotropy (or less anisotropy), with $\frac{\mathrm{N}_{\text {iso }}}{\mathrm{N}}=0.9859$, and larger bulk modulus $\mathrm{B}=64.43 \mathrm{GPa}$ than those for CuInSe2. Similarly, AgGaS2 possesses a closer material structure to isotropy and larger bulk modulus B than those for AgGaSe2.

\begin{tabular}{|l|c|c|c|c|c|c|c|}
\hline & C11 & C12 & C44 & C13 & C33 & C66 & a \\
\hline CuInS2 & 83.7 & 54.4 & 34.5 & 54.8 & 84.5 & 33.9 & $\begin{array}{l}\text { (Krustok et } \\
\text { al., 2001) }\end{array}$ \\
\hline $\begin{array}{l}\text { (Pantea et al., } \\
\text { CuInSe2 }\end{array}$ & 71.0 & 45.3 & 45.5 & 45.3 & 63.3 & 47.4 & $\begin{array}{l}\text { (Kannan et } \\
\text { al., 2004) }\end{array}$ \\
\hline $\begin{array}{l}\text { Pantea et al., } \\
\text { 2009) }\end{array}$ & 87.9 & 58.4 & 24.1 & 59.2 & 84.5 & 30.8 & $\begin{array}{l}\text { (Chahed et } \\
\text { al., 2005) }\end{array}$ \\
\hline $\begin{array}{l}\text { AgGaS2 } \\
\text { (Grimsditch \& } \\
\text { Holah, 1975) }\end{array}$ & 89.8 & 65.7 & 21.7 & 45.1 & 63.3 & 13.3 & $\begin{array}{l}\text { (Chahed et } \\
\text { al., 2005) }\end{array}$ \\
\hline $\begin{array}{l}\text { AgGaSe2 } \\
\text { (Eimerl et al., } \\
\text { 1991) }\end{array}$ & & & & & & & \\
\hline
\end{tabular}

Table 7. Elastic coefficients (GPa) and lattice constants a $(\mathrm{nm})$ for the hetrojunction layers compounds 


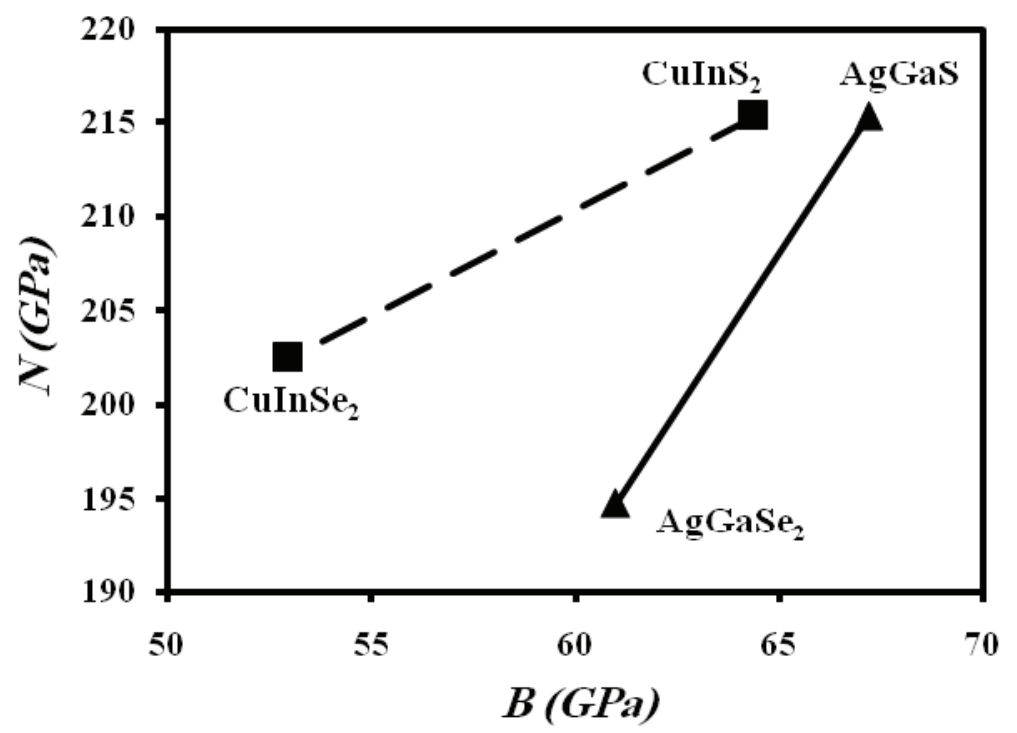

Fig. 4. The relation between the overall elastic constant $\mathrm{N}$ and bulk modulus $\mathrm{B}$ for the hetrojunction layer compounds

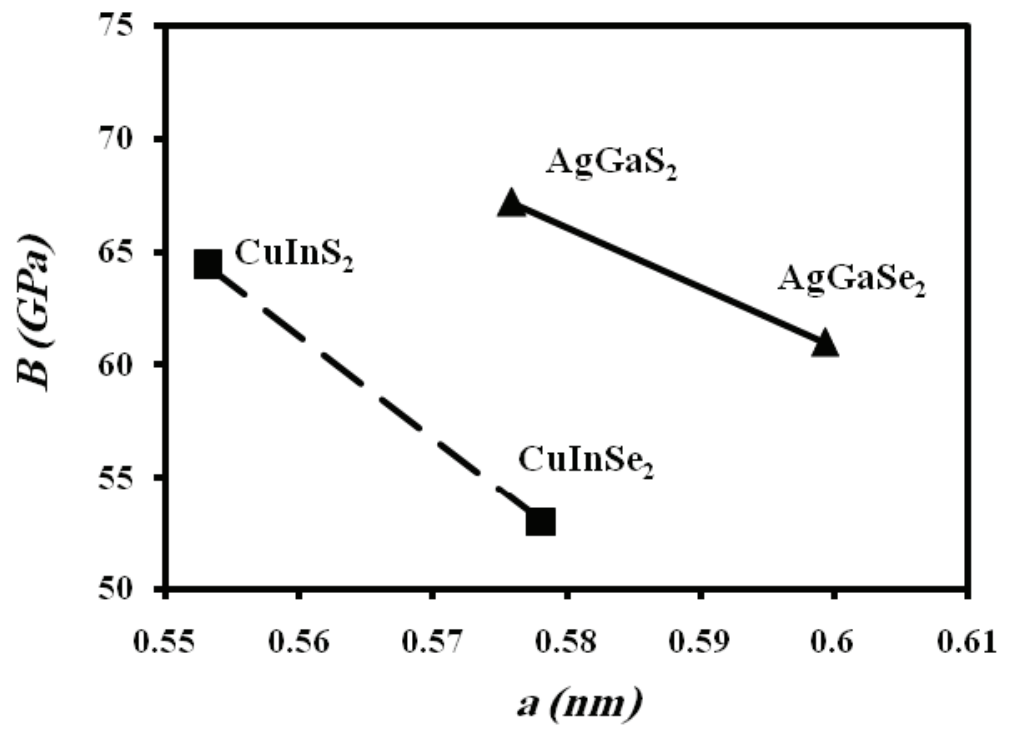

Fig. 5. The relation between the bulk modulus B and lattice constant a for the hetrojunction layer compounds 


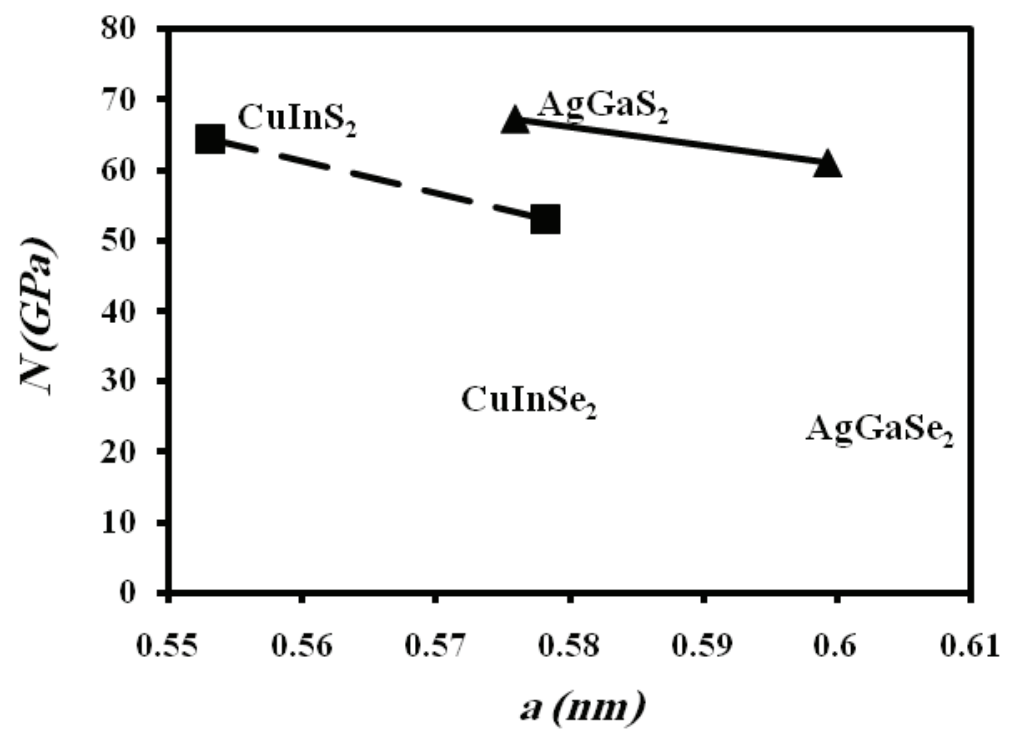

Fig. 6. The relation between the overall elastic constant $N$ and lattice constant a for the hetrojunction layer compounds

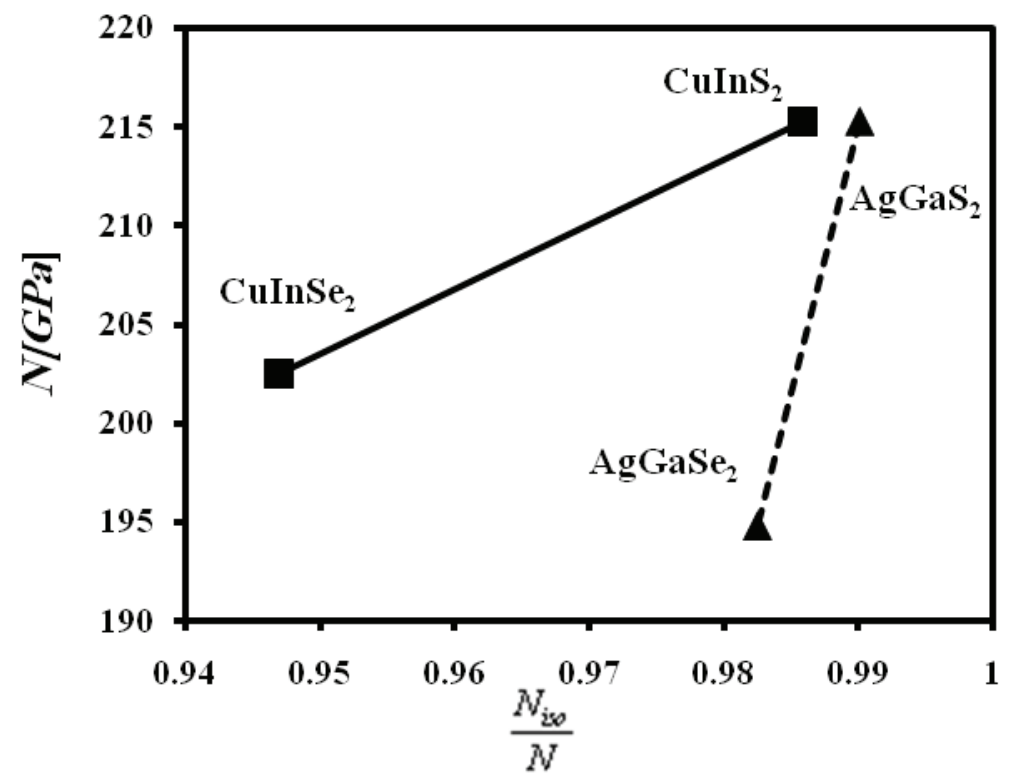

Fig. 7. The relation between the overall elastic constant $N$ and isotropy ratio $\frac{N_{i s o}}{N}$ for the hetrojunction layer compounds 


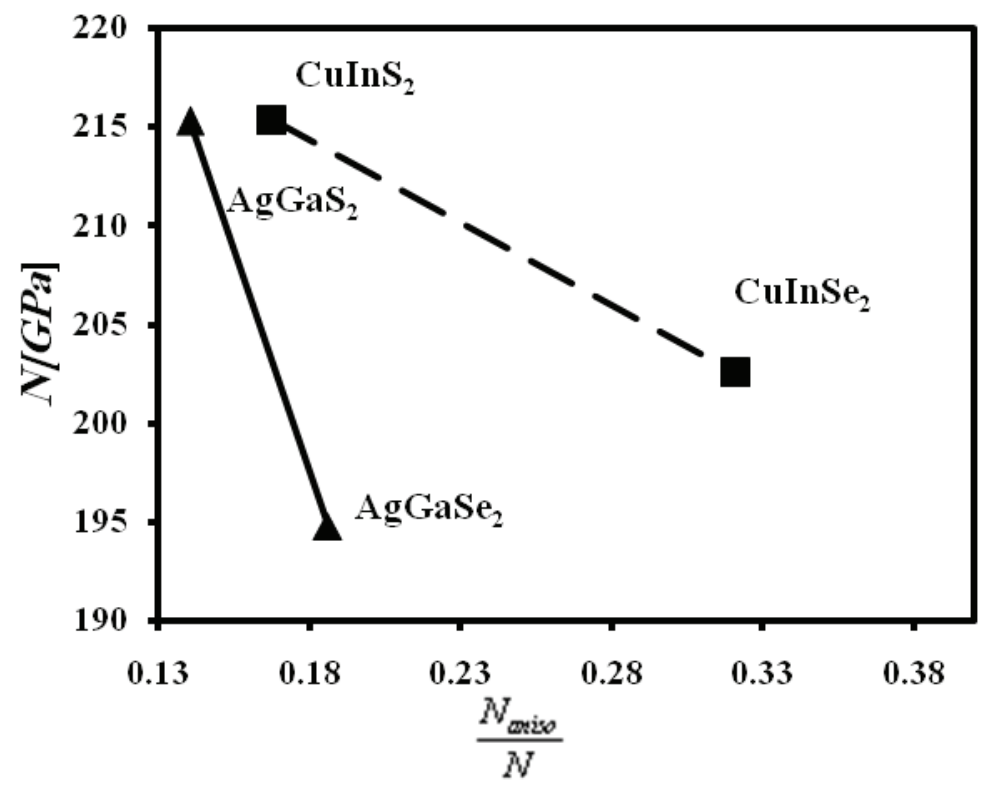

Fig. 8. The relation between the overall elastic constant $\mathrm{N}$ and anisotropy ratio $\frac{\mathrm{N}_{\text {anis }}}{\mathrm{N}}$ for the hetrojunction layer compounds

A significant contribution of this decomposition method is the direct correlation between the macroscopic and microscopic features of a material by means of symmetry properties. Based on the Orthonormal Tensor Basis Method (OTBM), the elastic stiffness for tetragonal system materials into two parts; isotropic (two terms) and anisotropic (four parts) is presented. The overall elastic stiffness is calculated and correlated with lattice constants and calculated bulk modulus for the hetrojunction layer compounds CuInSe2, CuInS2, AgGaSe2, and AgGaS2. The overall elastic stiffness is quantified and correlated to bulk modulus and inversely proportional to lattice constants. CuInS2 and AgGaS2 have larger overall elastic stiffness and bulk modulus and the smaller lattice constant than CuInSe2 and AgGaSe2, respectively. Based on the Norm Ratio Criteria (NRC), the hetrojunction layer compounds CuInS2 and AgGaS2 are closer to isotropy (or less anisotropic) than CuInSe2 and AgGaSe2.

\section{Conclusion}

Any physical property is characterized by $\mathrm{n}$ rank tensors, and this method is capable for decomposing these tensors with intrinsic symmetry, which is derived from the nature of the physical property itself, of any rank into orthonormal tensor basis. This method is capable to decompose tensors with non-intrinsic symmetry of rank $\mathrm{n}$ by generating an orthonormal basis using the well Known Gram-Schmidt process for the corresponding symmetry media of that tensor, and the number of basis elements should be equal to the number of nonvanishing distinct coefficients in that media. The decomposition procedure developed in this work has many engineering applications in anisotropic elastic materials which are, both 
qualitatively and quantitatively, different from isotropic materials. A new innovational decomposition of general and more explicit physical property for the symmetric second rank stress and strain tensors is introduced. The results are compared and found to be identical for special cases available in literature (Spencer, 1983; Cowin \& Mehrabadi, 1987; Hue \& Del Piero, 1991; Srinivasan, 1998; Blinowski \& Rychlewski, 1998; Tu, 1968; Nye, 1959; Ikeda, 1990). Nevertheless, this method is introducing a new form of decomposition that has a more featured and transparent physical information. The Criteria to measure the overall effect of the material properties proposed using the norms to represent the piezoelectricity and stiffness effect in the material like piezoceramics and fiber-reinforced composites, respectively. Through this method it is possible to study the effect of angle orientation of fibers and the material properties of fiber and matrix on the stiffness of the composite. A new proposed norm ratios criterion is introduced to measure the anisotropy degree and compare it with other materials of different symmetries. These ratios can be used to study the linear and non-liner damage parameters using total energy for fiber reinforced composite structures (will be published soon).

\section{References}

Al-Douri, Y.; Abid, H. \& Aourag, H. (2004). Empirical Formula Relating the Bulk Modulus to the Lattice Constant in Tetrahedral Semiconductors. Materials Chemistry and Physics, Vol. 87, pp. 14-17

Bätzner, D.L.; Romeo, A.; Terheggen, M.; Döbeli, M.; Zogg, H. \& Tiwari, A. N. (2004). Stability Aspects in CdTe/CdS Solar Cells. Thin Solid Films, Vol. 451, pp. 536-543

Blinowski, A. \& Rychlewski, J. (1998). Pure Shears in the Mechanics of Materials. Mathematics and Mechanics of Solids, Vol. 4, pp. 471-503

Chahed, A.; Benhelal, O.; Laksari, S.; Abbar, B.; Bouhafs, B.; Amrane, N. (2005). FirstPrinciples Calculations of the Structural, Electronic and Optical Properties of AgGaS2 and AgGaSe2. Physica B, Vol. 367, pp.142-151

Chelikowsky, J.R. (1987). High-Pressure Phase Transitions in Diamond and Zinc-Blende Semiconductors. Physical Review B, Vol. 35, pp. 1174-1180

Choi, In-Hwan, Yu, P.Y. (1996). Optical investigation of defects in AgGaS2 and CuGaS2. Journal of Chemistry and Physics of Solids, Vol. 57, pp.1695-1704

Cohen M.L. (1985) . Calculation of Bulk Moduli of Diamond and Zinc-Blende Solids. Physical Review B, Vol. 32, pp. 7988-7991

Cowin, S.C. \& Mehrabadi, M.M. (1987). On Eigentensors of Orthotropic Materials. Quarterly Journal of Mechanics and Applied Mathematics, Vol. 40, pp. 451-476

Eimerl, D.; Marion, J.; Graham, E. K.; McKinstry, H. A.; Haussuhl, S. (1991). Elastic Constants and Thermal Fracture of AgGaSe2 and d-Lap. IEEE Journal of Quantum Electrnics, Vol. 27, pp.142-145

Fischer, C.H.; Batzer, M.; Glatzel, T.; Lauermann, I., M. C.; Lux-Steiner, M. C. (2006). Interface Engineering in Chalcopyrite Thin Film Solar Devices. Solar Energy Materials \& Solar Cells, Vol. 90, pp. 1471-1585

Gaith, M. \& Akgoz, C.Y. (2005). A new Representation for the Properties of Anisotropic Elastic Fiber Reinforced Composite Materials. Review of Advance Material Sciences, Vol. 10, pp. 138-142 
Gaith, M. \& Alhayek, I. (2009). Correlation Between Overall Elastic Stiffness, Bulk Modulus and Interatomic Distance in Anisotropic Materials: Semiconductors. Review of Advance Material Sciences, Vol. 21, pp. 183-191

Grimsditch, N.S. \& Holah, G.D. (1975). Brillouin Scattering and Elastic Moduli of Silver Thiogallate (AgGaS2). Physical Review B, Vol. 12, pp. 4377-4382

Hearmon, R.F.S. (1961). An Introduction to Applied Anisotropic Elasticity. Oxford University Press

Hue, Y.Z. \& Del Piero, G. (1991). Journal elasticity. Vol. 21, pp. 203-246

Ikeda, T. (1990). Fundamentals of Piezoelectricity. Oxford University Press

Jerphagnon, J.; Chemla, D.S. \& Bonnevile, R. (1978). The Decomposition of Condensed Matter Using Irreducible Tensors. Advances in Physics, Vol. 27, pp. 609-650

Kannan, M.D.; Balasundaraprabhu, R.; Jayakumar, S. \& Ramanathaswamy, P. (2004). Preparation and Study of Structural and Optical Properties of CSVT deposited CuInSe2 thin films. Solar Energy Materials \& Solar Cells, Vol. 81, pp. 379-395

Kim E. \& Chen C. (2004). Calculation of Bulk Modulus for Highly Anisotropic Materials. Physics Letters A, Vol. 326, pp. 442-448

Krustok, J.; Raudija, J. \& Collan, H. (2001). Photoluminescence and the Tetragonal Distortion in CuInS2. Thin Solid Films, Vol. 387, pp. 195-197

Lam, P.K.; Cohen, M.L. \& Martinez, G. (1987). Analytic relation between bulk moduli and lattice constants. Physical Review B, Vol.35, pp. 9190

Leitsmann, R.L.; Ramos, L.E. \& Bechstedt, F. (2006). Structural Properties of PbTe/CdTe Interfaces from First Principles. Physical Review B, Vol. 74, pp. 085309

Murthy, Y.S.; Hussain O.M.; Naidu, S.B. \& Reddy, P.J. (1991). Characterization of pAglnSe2/n-Zn0.35Cd0.65S Polycrystalline Thin Film Hetrojunction. Materials Letters, Vol.10, pp. 504-508

Nowacki, W. (1962). Thermoelasticity. Oxford:Pergamon press

Nye, J.F. (1959). Physical Properties of Crystals; their Representation by Tensors and Matrices. Oxford: Clarendon Press

Nowacki, W. (1962). Thermoelasticity. Oxford:Pergamon press

Okuyama, H.; Miyajima, T.; Moringa, Y.; Hiei, F.; Ozawa, M. \& Akimot, K. (1992). Electronics Letters, Vol. 28, pp. 1798

Pantea, C.; Mihut, I.; Ledbetter, H.; Betts, J.B.; Zhao, Y.; Daemen, L.L.; Cynn, H. \& Miglori, A. (2009). Bulk Modulus of Osmium. Acta Materialia, Vol. 57, pp. 544-548

Radwan, F.A. (1991). Irreducible Cartesian Tensors in Anisotropic Continua. Ph.D. Thesis, METU, Turkey

Ramesh, P.P.; Hussain, O.M.; Uthanna, S.S.; Naidu, P. \& Reddy, P.J. (1998). Photovoltaic Performance of p-AgInSe2/n-Cds Thin Film Hetrojunction. Materials Letters, Vol. 34, pp. 217-221

Ramesh, P.P.; Hussain, O.M.; Uthanna, S.; Naidu, P.S. \& Reddy, P.J. (1999). Characterization of p-AglnSe2/n-Zn0.35Cd0.65S Polycrystalline Thin Film Hetrojunction. Materials Science and Engineering B, Vol. 49, pp.27-30

Reich, S.; Ferrari, A.C.; Arenal, R.; Loiseau, A.; Bellom, I. \& Robertson, J. (2005). Resonant Raman Scattering in Cubic and Hexagonal Boron Nitride. Physical Review B, Vol. 71, pp. 205201

Spencer, A.T.M. (1983). Continuum Mechanics, Longmans: London 
Srinivasan, T.P. \& Nigam, S.D. (1968). Invariant forms. Journal of Mathematical and Physical Sciences, Vol. 2, pp. 311-320

Srinivasan, T.P. (1969). Invariant Elastic Constants for Crystals. Journal of Mathematics and Mechanics, Vol. 19, pp. 411-420

Srinivasan, T.P. (1970). Invariant Piezoelectric Coefficients for Crystals. Phyica Staus Solidi, Vol. 41, pp. 615-620

Srinivasan, T.P. (1998). Decomposition of Tensors Representing Physical Properties of Crystals. Journal of Physics: Condens. Matter, Vol. 10, pp. 3489-3496

Tsai, S.C. (1992). Theory of Composite Design, Academic Press

Tu., Y.O. (1968). The Decomposition of Anisotropic Elastic Tensor. Acta Crystallographica A, Vol. 24, pp. 273-282

Voigt, W. (1889). The Relation Between the Two Elastic Moduli of Isotropic Materials. Annals of Physics, (Leipzig) Vol. 33, pp. 573 


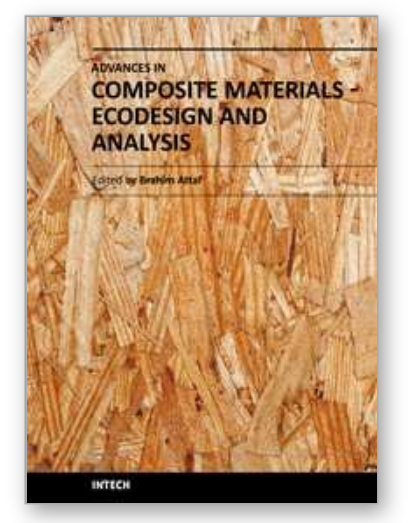

\author{
Advances in Composite Materials - Ecodesign and Analysis \\ Edited by Dr. Brahim Attaf
}

ISBN 978-953-307-150-3

Hard cover, 642 pages

Publisher InTech

Published online 16, March, 2011

Published in print edition March, 2011

By adopting the principles of sustainable design and cleaner production, this important book opens a new challenge in the world of composite materials and explores the achieved advancements of specialists in their respective areas of research and innovation. Contributions coming from both spaces of academia and industry were so diversified that the 28 chapters composing the book have been grouped into the following main parts: sustainable materials and ecodesign aspects, composite materials and curing processes, modelling and testing, strength of adhesive joints, characterization and thermal behaviour, all of which provides an invaluable overview of this fascinating subject area. Results achieved from theoretical, numerical and experimental investigations can help designers, manufacturers and suppliers involved with high-tech composite materials to boost competitiveness and innovation productivity.

\title{
How to reference
}

In order to correctly reference this scholarly work, feel free to copy and paste the following:

Mohamed S. Gaith and Imad Alhayek (2011). Estimation of the Degree of Anisotropy and Overall Elastic Stiffness of Advanced Anisotropic Materials, Advances in Composite Materials - Ecodesign and Analysis, Dr. Brahim Attaf (Ed.), ISBN: 978-953-307-150-3, InTech, Available from:

http://www.intechopen.com/books/advances-in-composite-materials-ecodesign-and-analysis/estimation-of-thedegree-of-anisotropy-and-overall-elastic-stiffness-of-advanced-anisotropic-materia

\section{INTECH}

open science | open minds

\section{InTech Europe}

University Campus STeP Ri

Slavka Krautzeka 83/A

51000 Rijeka, Croatia

Phone: +385 (51) 770447

Fax: +385 (51) 686166

www.intechopen.com

\section{InTech China}

Unit 405, Office Block, Hotel Equatorial Shanghai

No.65, Yan An Road (West), Shanghai, 200040, China

中国上海市延安西路65号上海国际贵都大饭店办公楼 405 单元

Phone: +86-21-62489820

Fax: $+86-21-62489821$ 
(C) 2011 The Author(s). Licensee IntechOpen. This chapter is distributed under the terms of the Creative Commons Attribution-NonCommercialShareAlike-3.0 License, which permits use, distribution and reproduction for non-commercial purposes, provided the original is properly cited and derivative works building on this content are distributed under the same license. 